\title{
Investigation of Transient Gas Phase Column Density Due to Droplet Evaporation
}

\author{
M.S. Woronowicz \\ Stinger Ghaffarian Technologies, Inc., 7701 Greenbelt Rd., Greenbelt, Maryland 20770 U.S.A. \\ michael.woronowicz@nasa.gov
}

\begin{abstract}
Analytical expressions are developed for estimating column density near a rapidly evaporating droplet along general paths. The influence for instantaneous evaporation is created first as a limiting case, where the peak value occurs at the time it takes a wave of vapor to reach the closest point along the optical path traveling at its most probable thermal speed. Next the case for finite-period evaporation is evaluated for constant conditions. Compared to the instantaneous case, peak column density occurs shortly after droplet extinction at a lower intensity. A new mathematical function is discovered that solves the integrals associated with this case. Finally, ways to account for droplet motion and changes in evaporation rate with size and temperature are discussed.
\end{abstract}

Keywords: column number density, plume flows, outgassing, free molecule flow PACS: $51.10+\mathrm{y}$

\section{INTRODUCTION}

Plans call for a NASA robotic refueling mission experiment on board the International Space Station (ISS) to repeatedly transfer a simulated cryogenic propellant between two dewars (Fluid Transfer Module, FTM). After each metered transfer, it is planned to vent that cryogen overboard via sublimation or evaporation. ISS payload providers must conduct analyses to demonstrate that any planned gaseous venting generate no more than a certain level of material that may interfere with optical measurements from other payloads that may be located nearby [1]. This requirement is expressed in terms of a critical maximum column number density (CND cit or $\sigma_{\text {crit }}$ ), with units of molecules $/ \mathrm{m}^{2}$. For the FTM venting process, it is likely that fluid droplets will accompany any such vapor.

Earlier related studies led to development of analytical expressions for $\sigma$ along general paths for a number of practical cases under steady conditions, including droplet evaporation [2]. These assumptions are violated for rapid evaporation of small droplets however, since they cannot sustain mass flow rates consistent with steady conditions.

In this work, analytical expressions are developed for estimating column density near a rapidly evaporating droplet along general paths. The influence for instantaneous evaporation is created first as a useful limiting case, where it is found the peak value occurs at the time it takes a wave of vapor to reach the closest point along the optical path traveling at its most probable thermal speed.

Next, a finite-period, constant-temperature evaporation case is evaluated. Compared to the instantaneous case, peak column density occurs shortly after droplet extinction, but at a lower intensity. A new mathematical function is discovered that solves the integrals associated with this case [3,4]. Finally, ways to account for droplet motion and changes in evaporation rate with size and temperature are discussed in this framework.

\section{MODEL DEVELOPMENT}

\section{Instantaneous Evaporation}

To describe gas being liberated from a point source with no directional constraints and no bulk velocity, this study uses a spherically-symmetric solution of the collisionless Boltzmann equation developed by Narasimha $[5,6]$. For the transient spatial evolution of number density $n$ in response to an instantaneous release of $N$ molecules, 


$$
n(r, t)=\frac{N \beta^{3}}{\pi \sqrt{\pi} t^{3}} e^{-\frac{\beta^{2} r^{2}}{t^{2}}} .
$$

Since the problem is spherically-symmetric, distance from the droplet center is given by radius $r$. Elapsed time is denoted by $t$, and parameter $\beta$ is the inverse of the most probable thermal speed $\sqrt{2 R T}$ [7], where $R$ is the speciesspecific gas constant and $T$ represents the droplet temperature.

Column number density $\sigma$ is given by the integrated effect of the vapor density intensity along some free path $l$ :

$$
\sigma=\int_{0}^{\infty} n d l
$$

where a general path from some initial location $r_{0}$ to the droplet source is depicted in Fig. 1 below.

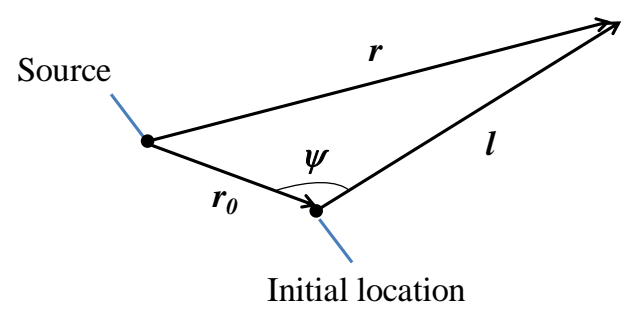

Figure 1. Geometry for evaluating $\sigma$ due to a radial point source.

In Fig. 1, the distance $r_{0}$ between the source and the path origin is fixed, as well as the path direction indicated by angle $\psi$, while $r$ and $l$ vary along the path. When substituting Eq. (1) into Eq. (2), one notes the only portion that is a function of $l$ is contained in the exponential factor. Let

$$
\xi \equiv \frac{\beta}{t} ; \quad \alpha_{0} \equiv \xi r_{0}
$$

Applying the Law of Cosines to relate $r$ to $l$, Eq. (2) becomes

$$
\sigma=\frac{N \beta^{3}}{\pi \sqrt{\pi} t^{3}} \int_{0}^{\infty} \exp \left[-\xi^{2}\left(r_{0}^{2}+l^{2}-2 l r_{0} \cos \psi\right)\right] d l .
$$

After some manipulation, one finds

$$
\sigma\left(r_{0}, \psi, t\right)=\frac{N \beta^{2}}{2 \pi t^{2}} e^{-\alpha_{0}^{2} \sin ^{2} \psi}\left[1+\operatorname{erf}\left(\alpha_{0} \cos \psi\right)\right]
$$


The solution for a radial path occurs when $\psi=\pi$, and the right-angle path $\sigma_{\perp}$ when $\psi=\pi / 2$. In Ref. 2 it was found that the maximum column density for any path was twice that of the right-angle path:

$$
\sigma_{\max }=2 \sigma_{\perp}=\frac{N \beta^{2}}{\pi t^{2}} e^{-\alpha_{0}^{2}}
$$

Eq. (6) may be differentiated to yield the time for peak column density to occur along this maximum path. The result is

$$
\left(t, \sigma_{\max }\right)_{\text {peak }}=\left(\beta r_{0}, \frac{N}{\pi e r_{0}^{2}}\right)
$$

Equation (7) indicates the peak column density along the path of maximum influence occurs when $\alpha_{0}=1$. As defined in Eq. (3), $\alpha_{0}$ represents a dimensionless wave velocity associated with thermal speed $1 / \beta$, which for a given species is completely governed by the droplet temperature.

Assuming a $d=1 \mathrm{~mm}$ droplet of methane $\left(8.33 \times 10^{18}\right.$ molecules at a liquid density of $\left.0.422 \mathrm{~g} / \mathrm{cm}^{3}\right)$ exited an FTM burst disk aperture at $150 \mathrm{~K}$ and evaporated instantaneously, column density levels for a radial path originating one meter away from the droplet's erstwhile position and the maximum influence path along $(-\infty, \infty)$ passing $1 \mathrm{~m}$ from that same position are presented in Fig. 2.

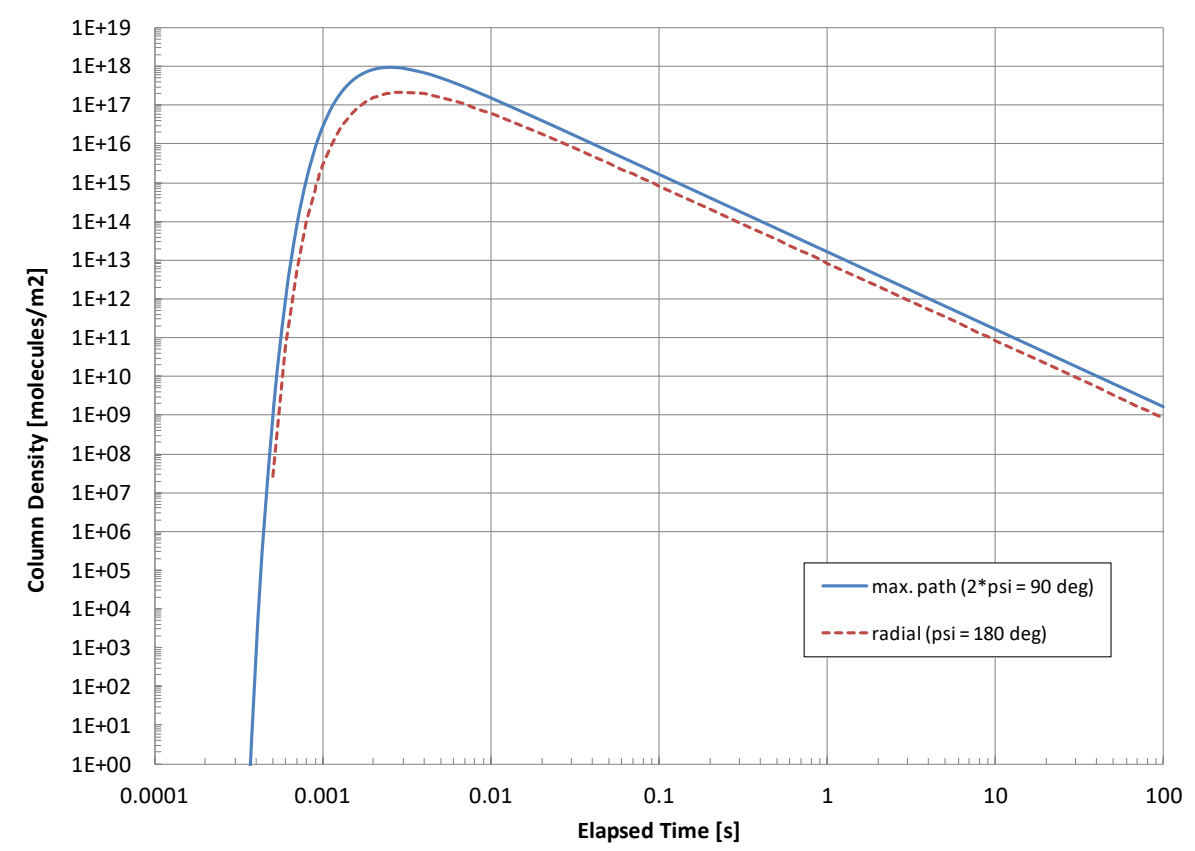

Figure 2. Transient column density, instantaneous evaporation of a $d=1 \mathrm{~mm} \mathrm{CH}_{4}$ droplet, $T=150 \mathrm{~K}$. 
For the maximum path, a peak value of $\sigma_{\text {peak }}=9.75 \times 10^{17}$ molecules $/ \mathrm{m}^{2}$ is found at $t=\beta r_{0}=2.53 \mathrm{~ms}$. As time increases, $\sigma \propto 1 / t^{2}$. For the path extending radially outward from $r_{0}=1 \mathrm{~m}$, the peak value is slightly lower, at $2.0 \times 10^{17}$ molecules $/ \mathrm{m}^{2}$, and occurs slightly later, at $3.0 \mathrm{~ms}$.

\section{General Peak Influence Condition}

Differentiating Eq. (5) with respect to time produces the following condition for the peak column density influence along a general path for $\pi / 2 \leq \psi \leq \pi$.

$$
\left(1-\alpha_{0}^{2} \sin ^{2} \psi\right)\left[1+\operatorname{erf}\left(\alpha_{0} \cos \psi\right)\right]=-\frac{\alpha_{0} \cos \psi}{\sqrt{\pi}} e^{-\alpha_{0}^{2} \cos ^{2} \psi}
$$

The radial path case $(\psi=\pi)$ simplifies to

$$
\operatorname{erfc} \alpha_{0}=\frac{\alpha_{0}}{\sqrt{\pi}} e^{-\alpha_{0}^{2}}
$$

While the maximum path peak condition occurs when $\alpha_{0}=1$, Eq. (9) satisfies the radial path case when $\alpha_{0} \approx 0.8420$. This behavior is reflected in Fig. 2 since $t \propto 1 / \alpha_{0}$

\section{Finite Evaporation Period}

The instantaneous evaporation limit should be considered a conservative approximation that produces worst-case peak values, but may underpredict the time to decay to some value if the peak violates the ISS constraint. In such cases it will be useful to describe the column density produced by a droplet that evaporates over a finite period.

For a rate that varies over time, one could approximate such behavior with a succession of discretized cases of instantaneous evaporation, each impulse representing the portion $\Delta N$ of the droplet of $N$ molecules that evolved over each time increment $\Delta t$. For constant-rate evaporation, $N=$ const., and Eq. (1) is replaced by

$$
n(r, t)=\int_{0}^{t} \frac{N \beta^{3}}{\pi \sqrt{\pi} t^{3}} e^{-\frac{\beta^{2} r^{2}}{t^{2}}} d t=\frac{\dot{N} \beta}{2 \pi \sqrt{\pi} r^{2}} e^{-\frac{\beta^{2} r^{2}}{t^{2}}} .
$$

Eq. (10) produces the correct steady limit for Narasimha's model,

$$
n(r, t \rightarrow \infty) \rightarrow \frac{\dot{N}}{\pi r^{2} \sqrt{8 \pi R T}}
$$

With a steady evaporation rate until some final extinction time $t_{f}, n(r, t)$ may be described by a square wave where Eq. (10) becomes the first of two nearly-identical terms, where the second replaces $t$ by $t-t_{f}$.

Finding the column density becomes a matter of solving Eq. (2) for these forms. Since these involve integrating over fixed path length $l$, the different time factors are of no consequence and one may focus attention on the "turnedon" period $\left(t \leq t_{f}\right)$. 
Substituting Eq. (10) into Eq. (2), applying the Law of Cosines to relate $r$ to $l$, and introducing $L \equiv l / r_{0}$ :

$$
\sigma\left(t \leq t_{f}\right)=\frac{\dot{N} \beta}{2 \pi \sqrt{\pi} r_{0}} e^{-\alpha_{0}^{2}} \int_{0}^{\infty} \frac{e^{-\alpha_{0}^{2}\left(L^{2}-2 L \cos \psi\right)}}{1+L^{2}-2 L \cos \psi} d L .
$$

\section{Right-Angle Case}

We shall concentrate attention first on the right-angle path column density $(\psi=\pi / 2)$ since it is half of the maximum influence solution.

$$
\sigma_{\perp}\left(t \leq t_{f}\right)=\frac{\dot{N} \beta}{2 \pi \sqrt{\pi} r_{0}} e^{-\alpha_{0}^{2}} \int_{0}^{\infty} \frac{e^{-\alpha_{0}^{2} L^{2}}}{1+L^{2}} d L=\frac{\dot{N} \beta}{2 \pi \sqrt{\pi} r_{0}} e^{-\alpha_{0}^{2}} I_{\perp} .
$$

The integrand in $I_{\perp}$ may be simplified by substituting $\eta \equiv \operatorname{Arctan} L$. Referring to Fig. 1, variable $\eta$ is the angle made between $r$ and $r_{0}$, and ranges from 0 to $\pi / 2$ as path length $l$ goes from zero to infinity.

$$
I_{\perp}=\int_{0}^{\pi / 2} e^{-\alpha_{0}^{2} \tan ^{2} \eta} d \eta
$$

It turns out a solution for Eq. (14) has not previously been identified. An approach recently developed by Woronowicz $[3,4]$ notes that integrals of the form

$$
I \equiv \int e^{f(\zeta)} d \zeta
$$

are solved by a function $H(\zeta)$ defined by

$$
H(\zeta) \equiv e^{-f(\zeta)} \int e^{f(\zeta)} d \zeta,
$$

which is the solution of the following differential equation:

$$
\frac{d H}{d \zeta}+H \frac{d f}{d \zeta}=1
$$

Applied to Eq. (14),

$$
\frac{d H}{d \eta}-2 \alpha_{0}^{2} \tan \eta \sec ^{2} \eta H=1
$$

Function $H$ is not particularly well behaved since it becomes unbounded as $\eta \rightarrow \pi / 2$ and rises steeply from zero when parameter $\alpha_{0}$ is sufficiently large. However, $\alpha_{0}$ (associated with inverse time) acts as a sort of switch that causes $H$ to change from $H \approx \eta$ for small values of $\alpha_{0}$ to $H \approx \exp \left(\alpha_{0}^{2} \sec ^{2} \eta\right)$ for large $\alpha_{0}$ and $\eta \rightarrow \pi / 2$. Figure 
3 illustrates how rapidly this switching occurs, with crossover characterized by conditions at $\log _{10} \alpha_{0}=0$, or $t=$ $r_{0} / \sqrt{2 R T}$.

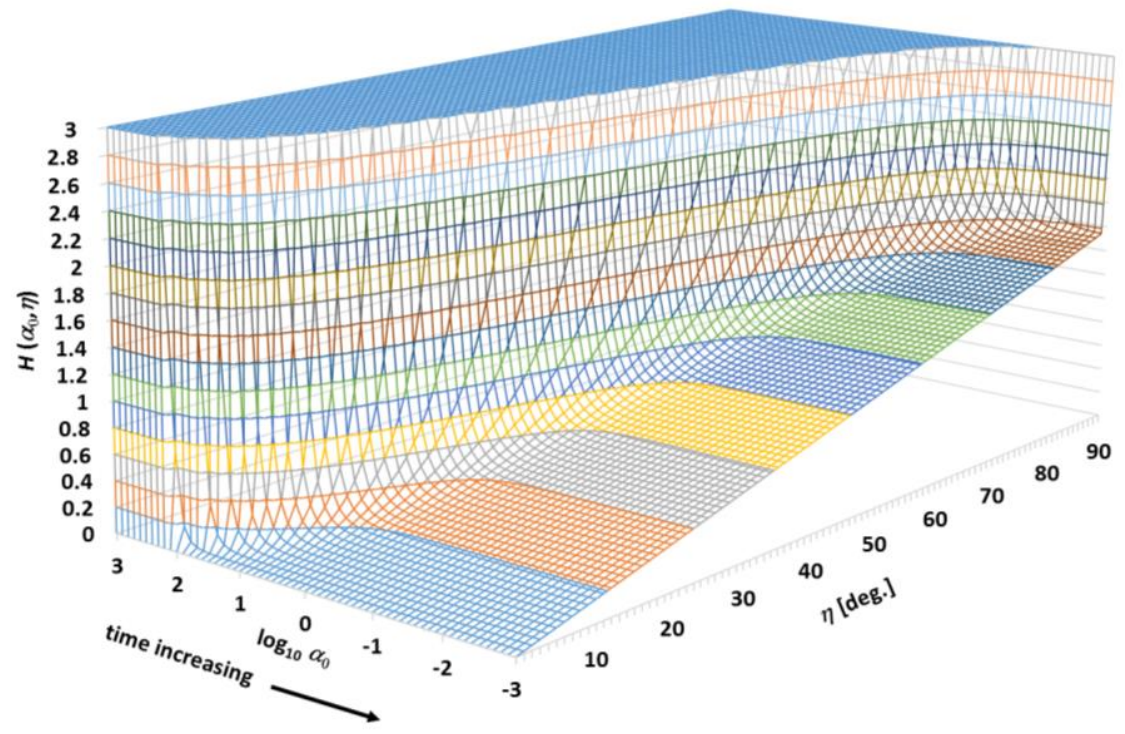

Figure 3. Three-dimensional contour map of $H\left(\alpha_{0}, \eta\right)$.

The conditions studied for instantaneous evaporation in the previous section are revisited in Fig. 4 to observe the impact of a finite evaporation period on the transient column density prediction. In actuality that period depends on the temperature-dependent evaporation rate, and that temperature also affects the most probable thermal speed (wave velocity $1 / \beta$ ), but this detail has been neglected to isolate the impact of evaporation period alone.

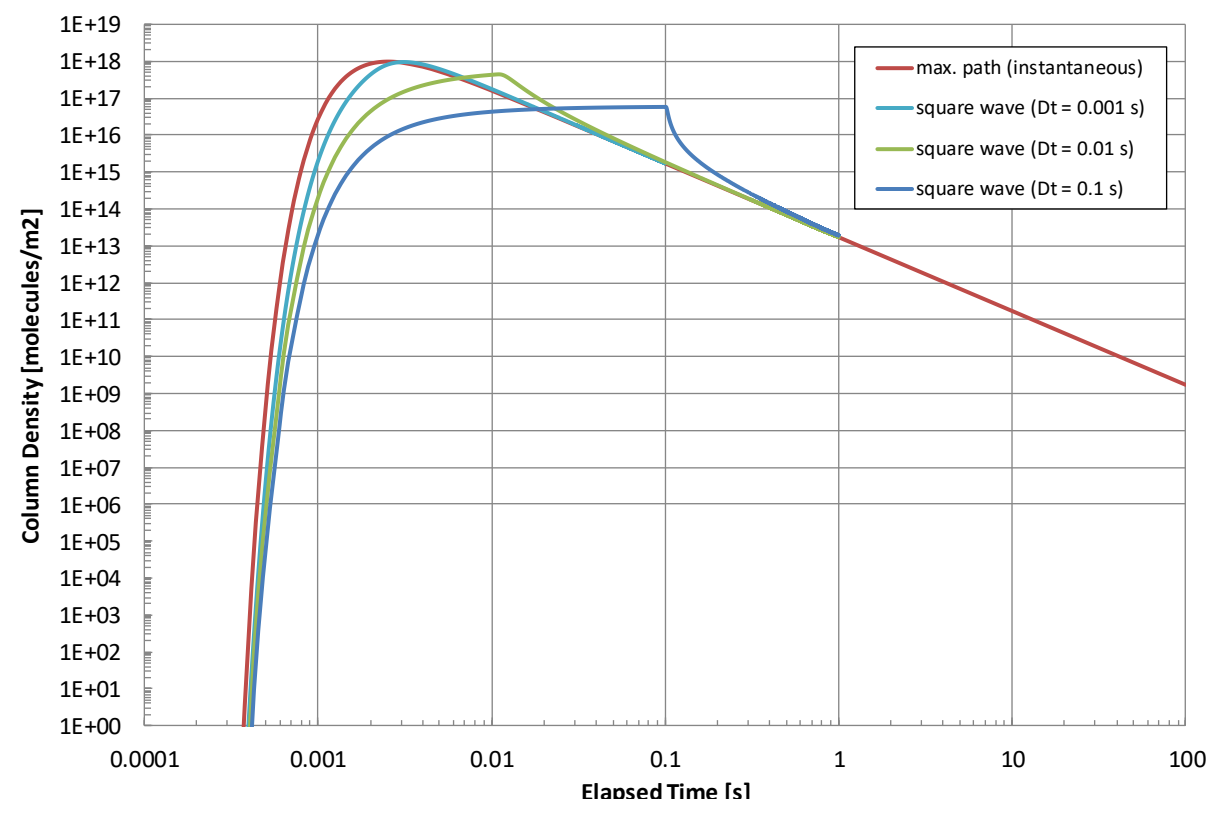

Figure 4. Transient column density, evaporation of a $d=1 \mathrm{~mm} \mathrm{CH}_{4}$ droplet over various periods, path of maximum influence, $T=150 \mathrm{~K}$ constant wave velocity. 
It is evident that the effect of a finite period is to reduce the peak value and to delay it to the end of the evaporation period when that time exceeds the peak effect for instantaneous evaporation. For brief evaporation periods, such as the $\Delta t=0.001 \mathrm{~s}$ run, the peak still occurs somewhat after that associated with the instantaneous limiting result, but quicker than simply adding $0.001 \mathrm{~s}$ to the time to reach the instantaneous evaporation peak. For all finite period cases considered in Fig. 4, the long term decay essentially settles to instantaneous-like behavior within ten evaporation periods, although that evolution takes more time with longer evaporation periods.

\section{General Case}

Returning to Eq. (12), the angle variable change analogous to the right-angle case becomes

$$
\eta \equiv \frac{1}{\sin \psi} \operatorname{Arctan}\left(\frac{L-\cos \psi}{\sin \psi}\right)
$$

producing

$$
\sigma\left(r_{0}, \psi, t\right)=\frac{\dot{N} \beta}{2 \pi \sqrt{\pi} r_{0}} e^{-\alpha_{0}^{2}\left(1+\cos ^{2} \psi\right)} \int_{\eta_{0}}^{\frac{\pi}{2} \csc \psi} e^{-\alpha_{0}^{2} \sin ^{2} \psi \tan ^{2}(\eta \sin \psi)} d \eta ; \quad \eta_{0} \equiv\left(\psi-\frac{\pi}{2}\right) \csc \psi
$$

To continue, one recognizes the radial case $\psi=\pi$ must be excluded. Creating a new variable $\gamma \equiv \eta \sin \psi$, the column density becomes

$$
\sigma\left(r_{0}, \psi, t\right)=\frac{\dot{N} \beta}{2 \pi \sqrt{\pi} r_{0}} \frac{e^{-\alpha_{0}^{2}\left(1+\cos ^{2} \psi\right)}}{\sin \psi} \int_{\psi-\frac{\pi}{2}}^{\frac{\pi}{2}} e^{-\tilde{\alpha}_{0}^{2} \tan ^{2} \gamma} d \gamma ; \quad \tilde{\alpha}_{0} \equiv \alpha_{0} \sin \psi
$$

Solution of the integral in Eq. (21) is satisfied by suitable applications of $H\left(\tilde{\alpha}_{0}, \eta\right)$.

Acute $\psi$ Path Modification

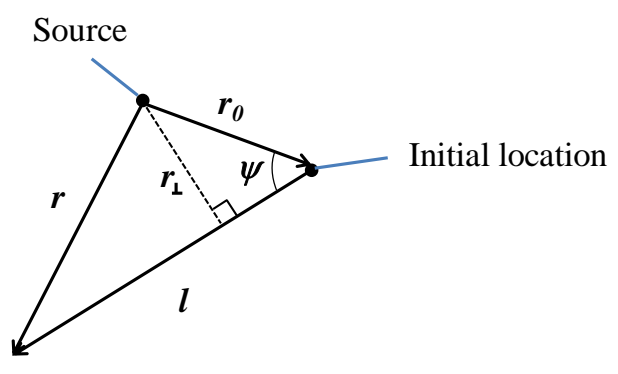

Figure 5. Geometry for $\psi<\pi / 2$ column density path.

For optical paths $l$ characterized by an acute angle $\psi$ (Fig. 5), $\sigma$ is given by the maximum path case where $r_{0}$ is replaced by $r_{\perp}=r_{0} \sin \psi$, minus a general case solution where $r_{0}$ is retained but $\psi$ is replaced by $\pi-\psi$. 
It has been noted that droplet or crystal temperatures tend to fall somewhat upon vacuum exposure [8]. That change affects the evaporation or sublimation rate $\dot{N}$ as well as the characteristic wave velocity $1 / \beta$. The fact that these droplets are in motion relative to a fixed optical path will also affect column density. All these effects may be compensated for approximately by defining how $r_{0}, \psi$, and $T$ vary with time relative to the optical path, and employ an incremental series of instantaneous releases to assess the overall impact. Computationally it would be straightforward to extend the effect of a single droplet to multiple droplets assuming negligible coupling between individual sources [9].

\section{CONCLUDING REMARKS}

A number of increasingly complex analytical expressions have been developed to assist investigators in describing the effect of transient single-droplet evaporation on column density along general paths, particularly for the path of maximum influence for a given separation distance between the droplet and the line of sight. The instantaneous evaporation case produces a limiting influence that may be used to quickly bound such effects given specific applications.

Moreover, column density solutions for droplets evaporating over finite periods were developed. This exploration lead to discovery of a new mathematical function that solves the integral of influence along the optical

path. For such cases, peak values occur shortly after extinction and at reduced values compared to the instantaneous case.

Finally, incorporation of further refinements including direct and indirect effects of transient temperature variation and motion were briefly discussed.

\section{ACKNOWLEDGMENTS}

MSW gratefully acknowledges support from NASA Contract NNG17CR69C, esp. Ms Kristina Montt de Garcia, NASA-GSFC Code 546, and Dr. Nora Bozzolo, Mr. Raymond LeVesque, and Dr. Dong-Shiun Lin, SGT, Inc.

\section{REFERENCES}

1. Attached Payload Interface Requirements Document, International Space Station Program, SSP 57003, Rev. H, p. 3-61, October 2011.

2. M.S. Woronowicz, in Proceedings of the $30^{\text {th }}$ International Symposium on Rarefied Gas Dynamics, American Institute of Physics, Melville, NY, 2016, pp. 170002-1-170002-8.

3. M.S. Woronowicz, New Mathematical Functions for Vacuum System Analysis, NASA CR-2017-219017, 2017.

4. M.S. Woronowicz, Some General Properties for a Newly Discovered Class of Mathematical Functions, NASA CR-2018-219028, 2018.

5. R. Narasimha, "Collisionless Expansion of Gases Into Vacuum," J. Fluid Mech., 12, pp. 294-308 (1962).

6. M.S. Woronowicz, in Proceedings of the $26^{\text {th }}$ International Symposium on Rarefied Gas Dynamics, American Institute of Physics, Melville, NY, 2009, pp. 565-570.

7. Vincenti, W., and Kruger, C., Introduction to Physical Gas Dynamics (Robert E. Krieger Publishing Company, Malabar, FL, 1965), p. 47.

8. Shin, et al., "Spherical-shaped ice particle production by spraying water in a vacuum chamber," Applied Thermal Engineering, Vol. 20, pp. 439-454, 2000.

9. Smith, et al., "Raman Thermometry Measurements of Free Evaporation from Liquid Water Droplets," $J$. American Chemical Society, Vol. 128, pp. 12892-12898, 2006. 